\title{
Grasping Objects by Their Handles: A Necessary Interaction Between Cognition and Action
}

\author{
Sarah H. Creem and Dennis R. Proffitt \\ University of Virginia
}

\begin{abstract}
Research has illustrated dissociations between "cognitive" and "action" systems, suggesting that different representations may underlie phenomenal experience and visuomotor behavior. However, these systems also interact. The present studies show a necessary interaction when semantic processing of an object is required for an appropriate action. Experiment 1 demonstrated that a semantic task interfered with grasping objects appropriately by their handles, but a visuospatial task did not. Experiment 2 assessed performance on a visuomotor task that had no semantic component and showed a reversal of the effects of the concurrent tasks. In Experiment 3, variations on concurrent word tasks suggested that retrieval of semantic information was necessary for appropriate grasping. In all, without semantic processing, the visuomotor system can direct the effective grasp of an object, but not in a manner that is appropriate for its use.
\end{abstract}

Dissociations between cognition and action have been proposed in several domains, including perception (Gibson, 1979), attention (Mack \& Rock, 1998), and the neural substrate of the visual system (Jeannerod, 1997; Milner \& Goodale, 1995). It has been repeatedly demonstrated that phenomenal experience and visuomotor behavior reflect differences that are suggestive of a separation in the representations underlying each. Neuropsychological studies indicate dissociations between object discrimination and visually guided action. For example, one patient may fail to discriminate between two different-sized objects, but her grasp of the object conforms to its size (Goodale, Milner, Jakobson, \& Carey, 1991). In contrast, another patient may discriminate objects accurately but is impaired in regard to reaching and grasping (Jakobson, Archibald, Carey, \& Goodale, 1991). Similarly, behavioral studies indicate that normal people may perceive a hill to be steeper than it actually is, but a motoric response directed at the hill is made accurately (Proffitt, Bhalla, Gossweiler, \& Midgett, 1995).

Although clear evidence for functional dissociations exists, it is also the case that cognition can influence action. Studies have shown that both where and when an action occurs influence whether the motoric response remains independent from cognition. A visual agnosic patient must direct her actions toward the object for accurate performance. Although the patient's grasp is effective when directed at an object, manual estimations to the side of the

Sarah H. Creem and Dennis R. Proffitt, Department of Psychology, University of Virginia.

This research was supported by National Institute of Mental Health Grant MH52640, National Aeronautics and Space Administration Grant NCC2925, and Defense Advanced Research Projects Agency Grant 539689-52273. We thank Mackenzie Carpenter. Chad Fawcett, Jarrod Markley, Victoria Nelson, Andrew Snyder, and Jessica Stockwell for assistance in data collection and coding.

Correspondence concerning this article should be addressed to Sarah $\mathrm{H}$. Creem, who is now at the University of Utah, Department of Psychology, 380 South 1530 East, Room 502, Salt Lake City, Utah 84112. Electronic mail may be sent to sarah.creem@psych.utah.edu. object do not correspond to the object's dimensions. Both motor responses are actions; however, only those actions directed at the object are accurate (Goodale et al., 1991). In addition, it has been shown in both normal and patient populations that, even when actions are directed toward objects, the introduction of a short time delay between viewing a stimulus and acting can induce a perceptual bias (Bridgeman, Peery, \& Anand, 1997; Creem \& Proffitt, 1998; Goodale, Jakobson, \& Keillor, 1994).

In the present studies, we show that cognition can influence actions even when the action is directed at the object and there is no delay in responding. Our action task was picking up familiar hand tools. Using a dual-task paradigm to interfere with cognitive processing, we show that people can pick up a hand tool using the visuomotor system alone, but they do so in a manner that is inappropriate for its use. For example, although people typically pick up a toothbrush by its handle rather than by its bristles, when cognitive processing is taxed, people will pick up a toothbrush by its bristles as readily as by its handle.

\section{Dissociable Systems?}

Evidence for the classification of separate processes for cognition and action within the visual system comes from a growing body of research on both neuropsychological patients and normal participants. Within neuropsychology, classic double dissociations in patient case studies support the existence of anatomically and functionally separate visual processing streams. Considerable research has been conducted with the visual form agnosic patient DF, who suffers from damage to her occipital-temporal lobe (see Milner \& Goodale, 1995, for a review). Although she has severely reduced conscious perception of form, she is able to guide her actions effectively to visually presented objects. In contrast, optic ataxic patients with posterior parietal damage are able to recognize and discriminate between objects, but they encounter a great deal of difficulty when attempting to guide their actions toward objects (Jakobson et al., 1991; Jeannerod, Decety, \& Michel, 1994).

Similarly, dissociations between visually guided action and phenomenal perception have been shown in a number of studies with 
normal participants. Using size illusions, researchers have illustrated that people's awareness of an object's size may be biased, whereas actions made toward the object are accurate (Aglioti, DeSouza, \& Goodale, 1995; Haffenden \& Goodale, 1998; Wraga, Creem, \& Proffitt, 2000; but see Franz, Gegenfurtner, Bulthoff, \& Fahle, 2000, and Pavani, Boscagli, Benvenuti, Rabuffetti, \& Farne, 1999, for alternative findings). Other studies have revealed contextual influences on a verbal perceptual response but not on a visually guided pointing response (Bridgeman et al., 1997). For example, Bridgeman et al. found that awareness of target position was influenced by the context of a surrounding frame, but pointing responses were not. In addition, systematic biases found in perceiving the slants of hills are not apparent in motoric adjustments related to the hills (Proffitt et al., 1995).

\section{When Is Action Informed by Cognition?}

Apparent dissociations between cognition and action occur only under limited circumstances. In other situations, interactions between these systems are evident. A critical factor in this regard is that a visually guided action is independent of perceptual representation only when it is directed at the stimulus. When presented with illusion-inducing patterns, such as the Ebbinghaus or MüllerLyer illusion, people can make manual size estimations by adjusting their thumb and index fingers to the size of the stimulus. This motoric response reflects the illusion. Conversely, when their grasp is directed toward the stimulus itself, there is no illusory bias (Haffenden \& Goodale, 1998; Welch, Post, Lum, \& Cohen, 1996). This distinction can also be seen in neuropsychological patients. Whereas the visual agnosic patient DF can grasp an object accurately, she cannot match the size of the object with her fingers (Goodale et al., 1991). A second important factor determining whether an action is influenced by cognitive representations is the time between viewing a stimulus and initiating a relevant action. Recent studies have shown that when time delays are introduced, the action system must rely on phenomenal awareness. In cases in which perceptual biases are involved, actions come to be influenced by the perceptual bias after a time delay (Bridgeman et al., 1997; Creem \& Proffitt, 1998; Rossetti, 1998). For example, Creem and Proffitt (1998) found that with a very short time delay, perceptual judgments (verbal) of a hill's slant were overestimated, whereas motoric estimates were not. However, after a longer delay, both verbal and motoric judgments increased proportionately in memory.

In patients, necessary interactions between the systems have been shown in a number of ways. The limitations to DF's visuomotor abilities suggest the circumstances under which information from cognition is needed. DF's abilities to guide her actions accurately include posting a card into slots of different orientations and grasping blocks of different widths (Goodale et al., 1991). Her visuomotor system can also adjust to variations in size and orientation of objects concurrently when the object has only one principal axis (Carey, Harvey, \& Milner, 1996). However, her ability fails when she must grasp a cross-shaped object (Carey et al., 1996) or post a T-shaped object into a slot (Goodale, Meenan, et al., 1994). Furthermore, whereas DF can guide a card into a slot defined by luminance boundaries, she cannot post the card into a slot defined by patterns based on Gestalt principles (Goodale, Jakobson, Milner, et al., 1994). This failure suggests that the visuomotor system alone may not be capable of using more subtle perceptual cues to guide action and must rely on the cognitive system for perceptual boundary cues such as similarity and proximity. Dijkerman, Milner, and Carey (1998) also showed recently that DF cannot guide three fingers of one hand into a disk with three holes. Dijkerman et al. suggested that this task requires allocentric coding that her intact action stream is incapable of processing.

All of these studies suggest that the "cognitive" system may be needed to guide actions toward more complex stimuli such as those defined by multiple principal axes, patterns, and nonegocentric frames of reference. Recent case studies also suggest instances of necessary interaction through evidence that patients can use their intact system to supplement their impaired system. Optic ataxic patient AT could not accurately grasp a neutral object such as a small cylinder. However, her grasping became much improved when the object was replaced with a familiar cylindrical object such as a tube of lipstick (Jeannerod et al., 1994). In this case, she used her intact cognitive system to identify the object and to inform her impaired motor guidance system.

\section{Overview of Studies}

The following studies demonstrate a necessary interaction between cognitive and motor systems using the visuomotor task of picking up hand tools. We propose that one can grasp an object effectively without the input of the cognitive system. However, directing a grasp that is appropriate to an object's use requires semantic processing by the cognitive system to inform the motor system about where and how to grasp the object. Our studies provide further evidence for both the separability and interactivity of the cognitive and visuomotor systems. In three studies, we used a dual-task interference paradigm to create specific processing deficits in normal participants. In Experiment 1, participants grasped hand tools while performing a concurrent, semantically challenging task. In this condition, the tools were typically not grasped in an appropriate manner by their handles. In contrast, when participants were given a concurrent visuospatial task that did not tax their semantic system, or when they were given no concurrent task at all, they grasped the objects appropriately. In Experiment 2, we assessed performance on a purely visuomotor task that had no semantic component. Here we found a reversal of results; the concurrent spatial task impaired visuomotor performance, but the semantic task did not. Experiment 3 further examined the nature of the interference present in Experiment 1. Varying the concurrent verbal task, we found that an easier semantic task interfered with handle grasping but that a nonsemantic verbal task did not.

In all of our grasping studies, participants were never explicitly told to pick up objects in a meaningful way. We began from the baseline finding that, if asked to grasp a tool, participants would spontaneously reach for the handle. It is likely that if they were explicitly told to pick up the objects by their handles in the dual-task conditions, they would continue to do so. We do not argue here that people fail to recognize the object during certain distractor tasks; instead, we suggest that they do not retrieve and process the object's functional characteristics to the same extent as when performing other tasks or no concurrent task at all. Although certain neuropsychological patients may fail to pick up objects 
appropriately when explicitly asked to use them, we do not expect such a severe deficit in neurologically intact participants. Thus, after having established that people do spontaneously grasp tools by their handles without explicit instruction, our intent was to measure the reduction in spontaneous appropriate grasping in dual-task conditions.

\section{Experiment 1}

\section{Method}

Participants. Twenty-eight University of Virginia undergraduate students ( 18 women and 10 men) participated in the experiment to satisfy a course requirement.

Materials. Ten different objects with distinct handles were used: comb, fork, hammer, knife, ladle, paintbrush, razor, spatula, screwdriver, and toothbrush. Participants were videotaped in all conditions with a Sony Hi8 video camera.

Design. Task condition was a between-subjects variable. Participants performed in the control $(n=9)$, semantic dual-task $(n=9)$, or spatial dual-task $(n=10)$ condition and were tested individually.

Procedure. In the principal task, grasping familiar objects, participants sat at a 3-ft-square (1-m-square) table. A blank sheet of paper $(8.5 \times 11$ in. $[21.6 \times 27.9 \mathrm{~cm}])$ was placed on the table on the side of the participant's dominant hand, about $8 \mathrm{in}$. from the participant's midline and $3 \mathrm{in}$. from the close edge of the table. Objects were placed individually on the table in front of the participant in one of three orientations. The handle always pointed away from the observer. The objects were placed vertically in front of the participant or rotated $45^{\circ}$ to the left or right of vertical. The experimenter always held the object at its center while placing it on the table. The center of the object was placed approximately 8 in. in front of the participant. The participant was instructed to pick up the object with his or her dominant hand and place it on the piece of paper. As soon as the object was placed on the paper, a new object was placed in front of the participant by the experimenter. Each object was presented twice for a total of 20 trials. In the control condition, participants performed this task alone.

In the semantic dual-task condition, participants performed the grasping task while completing a concurrent paired-associates distractor task. The task began with a training session before participants saw the objects. In the training session, participants heard 30 word pairs through headphones and then were tested on a paper-and-pencil task; they were given the first word of the pair and asked to fill in the second word. They repeated this process until they learned the word pairs to a criterion of $70 \%$ correct. The pairs consisted of words that were members of the same broad semantic category, such as "food" (see the Appendix for the word list). After training, they performed the testing phase of the paired-associates task while picking up the objects. They listened to a tape that presented the first word of every pair followed by a 2 -s period of silence. They were instructed to say the second word of the pair during the silence. Participants were instructed to try to respond to every word, even if they had to guess, and to perform the grasping task at the same time. When the participant heard the first word, the first object was placed on the table, and the testing phase began.

In the spatial dual-task condition, participants performed a spatial imagery distractor task, based on a task devised by Brooks (1968), in which they were asked to imagine a block letter and classify the corners. First, the experimenter showed the participants a series of block letters printed on separate pieces of paper. They were instructed on how to perform the task and tested to confirm their understanding of the task. The participants were instructed to classify the corners of the letter by saying "yes" if the corners were on the top or bottom of the figure and "no" if the corners were anywhere else in the figure. They were to perform this task at the same time as the grasping task. The testing began when the experimenter named the first letter and then placed the first object in front of the participant. When the participant had finished classifying the corners of one letter. the experimenter immediately named the next letter so that the tasks remained simultaneous.

\section{Results}

Videotapes were coded to detcrmine whether grasping was appropriate for the object's use. Trials were scored with a $i$ if the grasp was appropriate and a 0 if it was not. Criteria for appropriateness were that the participant reached around and picked up the handle of the object. Because the handle always faced away. it score of 1 was not given if the participant simply reached forward for the handle or for another part of the object; it was necessary to reach around the object for the handle (see Figure 1). A total score
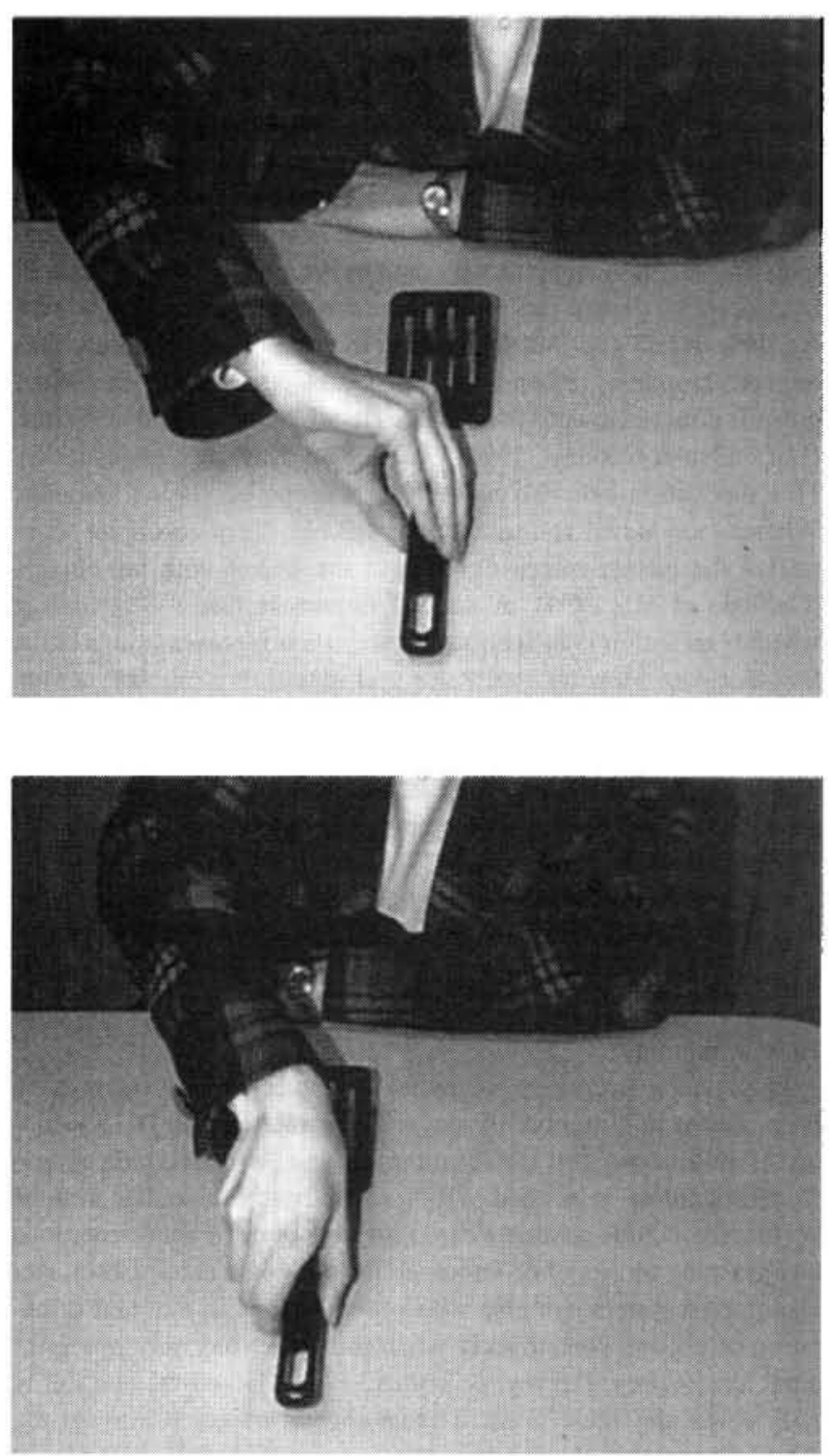

Figure 1. Top: A grasp coded as "correct" because the participant reached around to pick up the handle as if she were to use the object. Bottom: A grasp coded as "incorrect": although the participant grasped the handle. it was not an appropriate grasp for its use. 
with a maximum of 20 was given to each participant. Each participant's videotape was independently coded by one of the authors and a naive coder who was not aware of the predictions for each condition. The reliability ratio between the two sets of scores was $95 \%$.

Figure 2 presents percentages of appropriate grasps for each task condition. Log-odds ratios of appropriate to inappropriate grasps were calculated to avoid the skew of frequency counts (Tukey, 1977). The log-transformed scores were compared in a univariate analysis of variance (ANOVA) with three levels of task condition (control, semantic, or spatial). ${ }^{1}$ There was a significant effect of task condition, $F(2,25)=11.72, p<.001$. Planned simple contrasts revealed that appropriateness scores for the semantic dual-task group ( $M=17 \%$ ) were much lower than those for the control group $(M=72 \% ; p<.001)$. However, there was no difference between the spatial dual-task group $(M=55 \%)$ and the control group $(p<.12)$.

The influence of tool orientation was also evaluated for each condition. A 3 (task condition) $\times 3$ (orientation) mixed-design ANOVA was performed on percentage of appropriate grasps, with task condition as a between-subjects variable and orientation as a within-subject variable. Orientation of the objects was labeled as "towards" (handle closer to the dominant hand), "neutral" (handle pointing directly away from the observer), or "away" (handle farther from dominant hand). In all, more handles were grasped in the "towards" condition than in the other orientations. The analysis revealed a significant effect of orientation, $F(2,50)=49.36, p<$ .001 , as well as an Orientation $\times$ Task Condition interaction, $F(4$, $50)=6.17, p<.001$. Figure 3 indicates that, in all task conditions, appropriate grasping resulted most in the "towards" orientation, followed by the "neutral" and "away" orientations. Although this pattern of behavior was consistent across task conditions, the interaction indicated a reliable difference between the "towards" and "neutral" orientations in the spatial and semantic tasks but not in the control task.

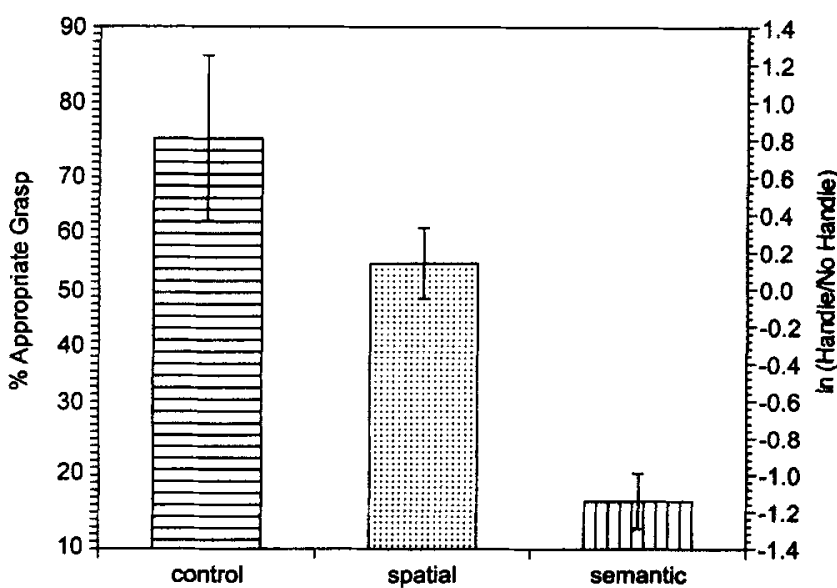

Figure 2. Mean percentages of objects grasped appropriately ( $\pm 1 S E$ ) for the control (grasping alone), spatial dual-task, and semantic dual-task conditions in Experiment 1 . The $y$-axis presents both percentage of appropriate grasps and the natural $\log (\mathrm{ln})$ of the ratio of "handle" to "no handle" responses.

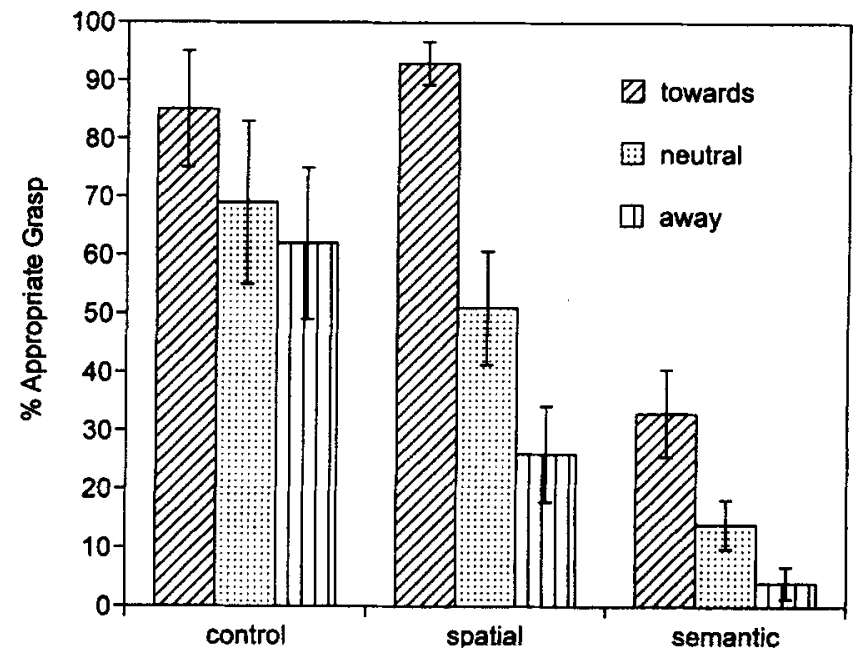

Figure 3. Mean percentages of objects grasped appropriately ( $\pm 1 S E)$ as a function of object orientation in Experiment 1 .

\section{Discussion}

When participants were asked to pick up objects without a secondary task, they most often reached around to pick the objects up by their handles in a manner appropriate for use of the objects. However, when the cognitive system was taxed by a concurrent semantic task, they rarely picked up the objects appropriately. The semantic task may have interfered with the depth to which participants could semantically process the objects and, thereby, limited the information made available to the visuomotor system about where to grasp the object. Furthermore, the results from the spatial dual-task condition suggest that the interference was specific to the semantic system responsible for identifying the object and its functional qualities. There was little decrement in performance when the spatial task was performed concurrently.

Differential effects of object orientation suggest that, without interference, participants generally picked up objects by their handles at similar frequencies regardless of the orientation. However, in the interference conditions, participants were more likely to grasp objects by the "near" orientation, which was a much less awkward grasp biomechanically. This was the result in both dualtask conditions; however, the overall degree to which objects were grasped appropriately at all was greatly reduced in the semantic dual-task condition.

One possible explanation for the apparent dissociation between dual-task effects is that the spatial and semantic secondary tasks were not equally distracting. It could be that the semantic task was inherently more difficult than the spatial task and thus interfered with appropriate grasping as a result of difficulty rather than specific processing demands. Another possibility is that there was a differential trade-off in performance between the grasping task

\footnotetext{
${ }^{1}$ Although log-transformed ratios of appropriate to inappropriate scores were analyzed, mean percentage appropriate scores are reported in the text for clarity of presentation.
} 
and each of the distractors. In other words, participants could have grasped more appropriately with the concurrent spatial distractor than with the semantic distractor because they performed diligently on the semantic task but less diligently on the spatial task. Experiment 2 addressed both of these possibilities by using a primary task that was a purely visuospatial motor task without a semantic component and comparing performance with both distractors. Using another visuomotor task, we were also able to compare performance on both distractors across both studies.

\section{Experiment 2}

To further examine the interaction between appropriate grasping and type of distractor that was illustrated in Experiment 1 , we used a visuomotor task that did not have a semantic identification component and tested performance with the two dual-task conditions. This task involved tracking a moving dot on a computer screen. It allowed us to determine that the impaired performance in Experiment 1 was not simply a result of depleted attentional resources or greater difficulty of the semantic task than the spatial task but, rather, a result of interference to a necessary semantic component of the grasping task. We predicted that tracking performance would not be impaired by the semantic inference task because the motor task did not involve meaningful processing of an object, as in the task of Experiment 1. In contrast, performance would be impaired with the concurrent spatial task, because tracking the dot did involve visuospatial processing.

\section{Method}

Participants. Thirty-four University of Virginia undergraduates (17 men and 17 women) participated in the experiment to satisfy a course requirement.

Design. All participants performed in both a control condition and one of the dual-task conditions, semantic or spatial.

Procedure. Participants performed a pursuit-tracking task (Willingham, Hollier, \& Joseph, 1995) on a Macintosh PowerPC. In the tracking task, the participant positioned a cross-hair cursor over a randomly linearly moving 1.06-cm-diameter target dot on a computer screen using a regular mouse. The target began moving at $105.6 \mathrm{~mm} / \mathrm{s}$ when the participant positioned the cursor over it and clicked the mouse. The target moved continuously in $40-\mathrm{s}$ blocks. The location of the cursor and the target dot was recorded every $200 \mathrm{~ms}$. Participants were given one 40 -s block of practice to become accustomed to the task. Then they performed two more blocks of the control task, followed by two blocks of one of the dual-task conditions.

The dual-task conditions were conducted similarly to those in Experiment 1 , except that the grasping task was replaced by the tracking task. Participants were instructed to try to perform to the best of their ability on both tasks at the same time. In the semantic dual task, participants learned the same word pairs to criterion as in Experiment 1. In the testing phase, they started responding to words as soon as they clicked on the target dot. In the spatial dual task, participants were first shown the block letters as in Experiment 1 . They began the letter classification as soon as they clicked on the dot.

\section{Results}

Tracking task. Analyses were performed on a measure of root mean square error (RMSE) calculated from the mean distance from the target to the cursor (in pixels) throughout the block, minus the first $5 \mathrm{~s}$. A 2 (task condition: control vs. dual task) $\times 2$ (sex) $\times 2$ (dual-task type: spatial vs. semantic) mixed-design ANOVA was performed on RMSE with task condition as a within-subject variable and sex and dual-task type as betweensubjects variables. The results revealed main effects of task condition, $F(1,29)=26.07, p<.001$, and dual-task type, $F(1$, $29)=21.31, p<.001$, and, most important, a Task Condition $\times$ Dual-Task Type interaction, $F(1,29)=18.44, p<.001$. Separate 2 (task condition) $\times 2$ (sex) ANOVAs for each dualtask type (spatial and semantic) were performed to assess the interaction. The interaction showed that whereas RMSE increased with the spatial dual task ( $M=40.08$ pixels) relative to the control task $(M=30.81), F(1,15)=25.16, p<.001$, there was no difference in RMSE between the semantic dual task $(M=29.33)$ and the control task $(M=28.52), F(1,14)=2.38$, $p<.145$ (see Figure 4).

Between-experiments distractor analysis. Performance on the secondary tasks was assessed to ensure that a trade-off in performance between the primary and distractor tasks was not the cause of the reversal of effects on the primary tasks. Percentage correct responses were calculated for both the semantic and spatial distractor tasks performed with the grasping task of Experiment 1 and the tracking task of Experiment 2 . The analysis revealed no differential performance on the semantic or spatial distractors as a function of the primary task performed. A 2 (primary task) $\times 2$ (secondary task) between-subjects ANOVA showed only an effect of secondary task type, $F(1,39)=19.50, p<.001$, illustrating that, overall, performance was better on the spatial task than on the semantic task regardless of the primary task performed (spatial: $M=85 \%$; semantic: $M=67 \%$ ). Figure 5 shows that there was no difference in percentage correct as a function of the primary task

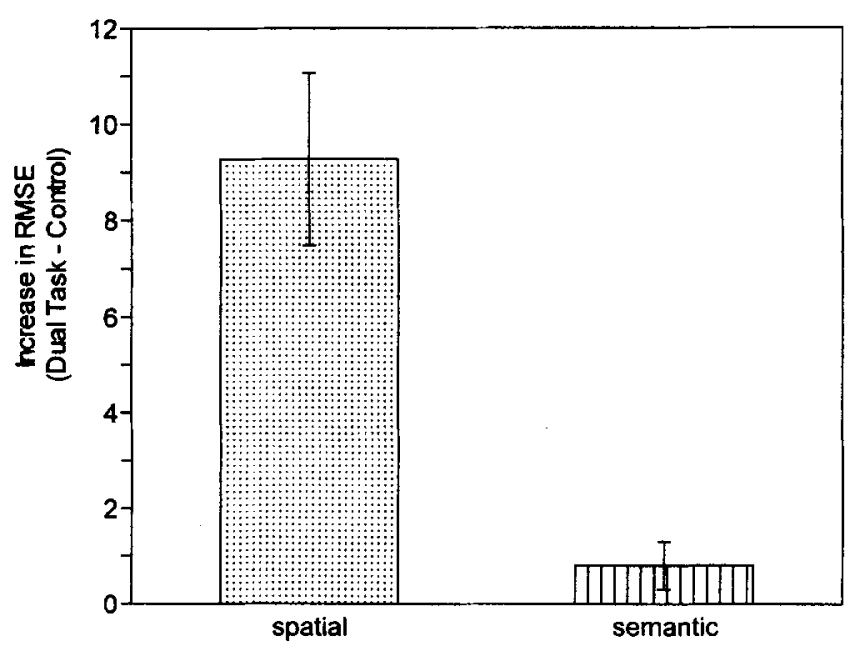

Figure 4. Mean increases in error rates $( \pm 1 S E)$ for the spatial and semantic dual-task conditions in Experiment 2, calculated as the difference between root mean square error (RMSE; pixels) for the dual task minus the control (tracking alone) task. 


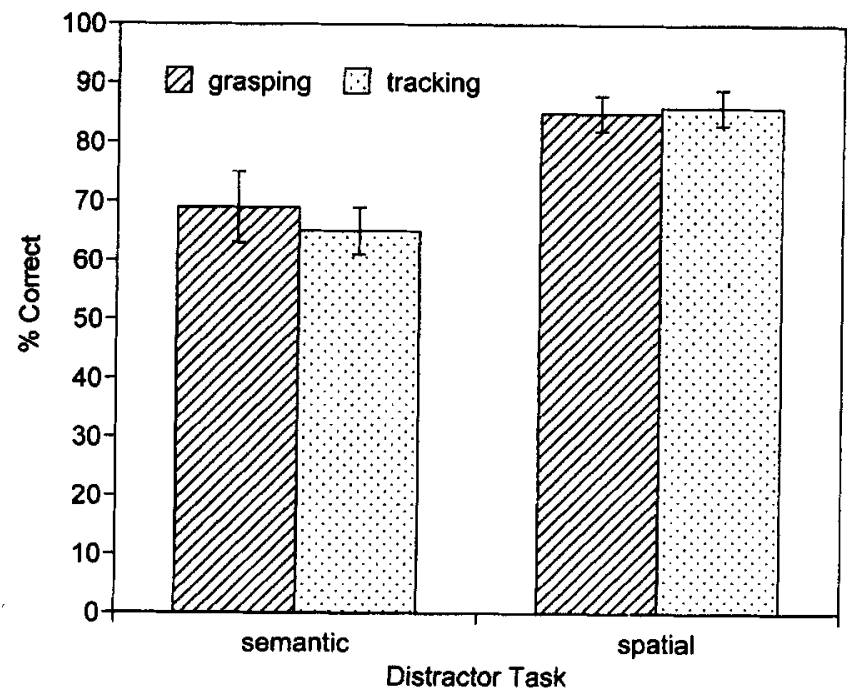

Figure 5. Mean percentages of correct responses $( \pm 1 S E)$ on semantic and spatial distractor tasks as a function of primary task performed.

(grasping or tracking) performed, $F(1,39)=0.16, p=.69$ (grasping: $M=77 \%$; tracking: $M=75 \%$ ). ${ }^{2}$

\section{Discussion}

In contrast to Experiment 1, the spatial dual task interfered with the visuomotor task, whereas the semantic dual task did not. These results are similar to those of Baddeley, Grant, Wight, and Thomson (1975), who found a decrement in visual pursuit tracking with a concurrent spatial task but not with a verbal task. The reversal of results between Experiments 1 and 2 supports our claim that grasping tools spontaneously by their handles requires semantic processing to inform the visuomotor response. In Experiment 2, the motoric task did not involve semantic processing and thus was not interfered with by the same task. We also found the reverse pattern for the spatial dual task. Whereas the spatial task did not interfere with the semantic component needed for grasping familiar objects, it did interfere with the tracking task that was necessarily spatially driven.

Furthermore, the between-experiments analysis allowed us to more confidently claim that the reversal of impairment in the primary tasks was not a result of trade-off in performance with the distractor task. Participants' performance on the secondary tasks was equivalent, regardless of the primary task performed. Thus, Experiments 1 and 2 illustrate a clear double dissociation. Appropriate grasping was impaired by the semantic distractor but not by the spatial distractor. In contrast, visuomotor tracking was impaired by the spatial distractor but not by the semantic one. We suggest that the specific interference of the semantic task in appropriate grasping implicates the cognitive (semantic) system as a necessary component in guiding actions appropriately.

\section{Experiment 3}

Although the reversal of dual-task effects in Experiment 2 suggests that it was not the differential difficulty of the two distractor tasks that led to differential interference effects in Ex- periment 1 , the question of defining the nature of the interference still remains. The paired-associates task in Experiment 1 that led to interference was semantic in nature but also involved a difficult memory component. Our aim in Experiment 3 was to create a less cognitively demanding semantic task to further assess the processes required for grasping objects appropriately by their handles. We created a free-associates task in which participants were presented with one word and were free to name any second word. In addition, we created two other variations of verbal tasks. Participants performed the free-associates task, a standard articulatory suppression task, or a paired-associates task involving nonsense syllables.

\section{Method}

Participants. Thirty University of Virginia undergraduates (12 men and 18 women) participated in the experiment to satisfy a course requirement.

Design. Task condition was a between-subjects variable. Participants performed in the free-associates $(n=10)$, nonsense-syllable $(n=10)$, or articulatory suppression $(n=10)$ task condition and took part individually.

Procedure. The primary grasping task was performed in the same manner as in Experiment 1. There were three variations of the concurrent task distractor.

For the free-associates distractor, participants performed in both a pretesting and testing session. In pretesting, they listened to a list of 30 words on audiotape. The list of words was composed of the first word of every word pair from Experiment 1. They were instructed to say any associated word immediately after hearing a word during a 2-s period of silence. The words were presented continuously throughout the experiment. In testing, participants listened to a new list of 30 words. The list was composed of the second word of each paired associate from Experiment 1. Participants were instructed to respond with any words, as in pretesting, and at the same time perform the grasping task of Experiment 1.

The second distractor task was the nonsense-syllable-pairs distractor. As in the paired-associates task of Experiment 1, in training, participants listened to a list of pairs of words and then were given a paper-and-pencil test to assess their knowledge of the syllable pairs. The word pairs consisted of 10 different pairs of consonant-vowel-consonant rhyming nonsense syllables (e.g., ZIJ-KIJ; see Appendix for syllable list). The training continued until participants reached a criterion of $80 \%$ correct. After training, they performed the testing phase of the paired-associates task while picking up the objects. They listened to a tape that presented the first syllable of every pair followed by a 2-s period of silence. They were instructed to say the second syllable of the pair, because another syllable would immediately follow. The syllable-pair list was repeated three times. The final distractor task was the articulatory suppression distractor. For this task, participants were instructed to repeat the word la continuously while picking up the objects.

\section{Results}

In all, the free-associates and nonsense-syllable task conditions showed a decrement in number of appropriate grasps, whereas the articulatory task condition showed equivalent performance to the control condition. Videotapes were coded in the same manner as in Experiment 1. A total score with a maximum of 20 was given to

\footnotetext{
${ }^{2}$ Data from 8 participants in the semantic-tracking condition were not recorded. The sample sizes analyzed for each group were as follows: semantic-grasping, $n=9$; semantic-tracking, $n=7$; spatial-grasping, $n=10$; and spatial-tracking, $n=18$.
} 
each participant. Each participant's videotape was independently coded by one of the authors and a naive coder who was not aware of the predictions for each condition. The reliability ratio between the two sets of scores was $96 \%$. The three experimental conditions were compared with the control condition of Experiment 1.

Figure 6 presents percentages of appropriate grasps for each task condition. Log-odds ratios of appropriate to inappropriate grasps were calculated to avoid the skew of frequency counts (Tukey, 1977). The log-transformed scores were compared in a univariate ANOVA with four levels of task condition (control, articulatory, nonsense syllables, and free associates). ${ }^{3}$ There was a significant effect of task condition, $F(3,35)=7.98, p<.001$. Planned simple contrasts between the dual-task conditions and the control condition revealed lower appropriateness scores for the free-associates task $(M=22 \% ; p<.001)$ and the nonsense-syllable task $(M=$ $31 \% ; p<.01)$ than for the control task $(M=72 \%)$. However, there was no difference between the articulatory task $(M=73 \%)$ and the control task $(p=.87)$.

As in Experiment 1, effects of object orientation were investigated. A 3 (orientation) $\times 3$ (task condition) mixed-design ANOVA was performed on percentages of appropriate grasps. The analysis revealed an overall effect of orientation, $F(2,54)=30.66$, $p<.001$, and no interaction with task condition $(p=.64)$. Handle-grasping percentages were greater in the "towards" than "neutral" orientation, $F(1,27)=28.44, p<.001$, and greater in the "neutral" than "away" orientation, $F(1,27)=13.92, p<.001$ (see Figure 7), across all conditions.

Furthermore, as in Experiments 1 and 2, performance on the distractor tasks themselves was assessed. Because there was no "correct" answer for the free-associates task, performance was assessed simply by percentage of coherent responses produced. The mean percentage of responses was $93 \%$. Performance on the nonsense-syllable task was $71 \%$ correct.

\section{Discussion}

Experiment 3 further investigated the nature of the interference seen in Experiment 1. Our main goal was to create a semantic

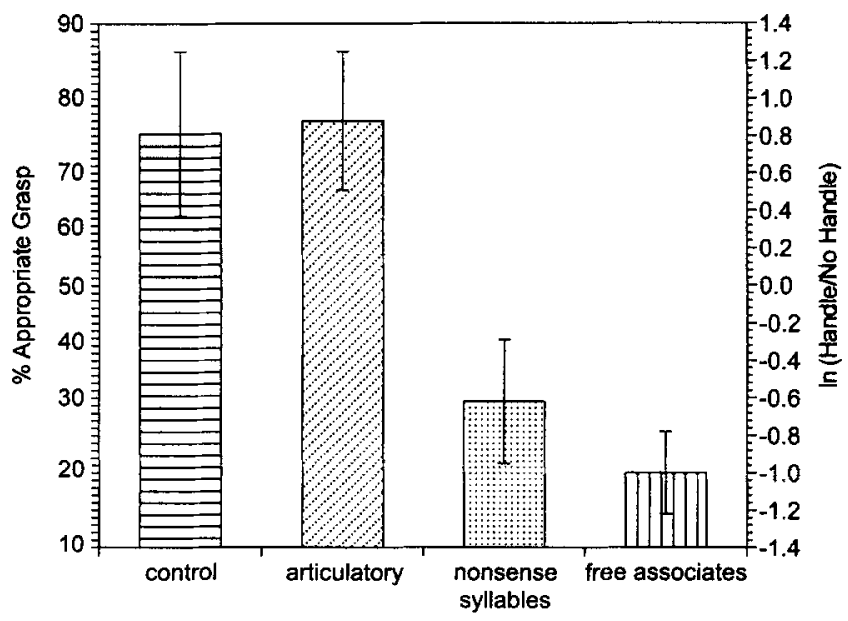

Figure 6. Mean percentages of objects grasped appropriately ( $\pm 1 S E$ ) for the control task (grasping alone) and the three verbal distractor tasks in Experiment 3 . The $y$-axis presents both percentage of appropriate grasps and the natural $\log (\ln )$ of the ratio of "handle" to "no handle" responses.

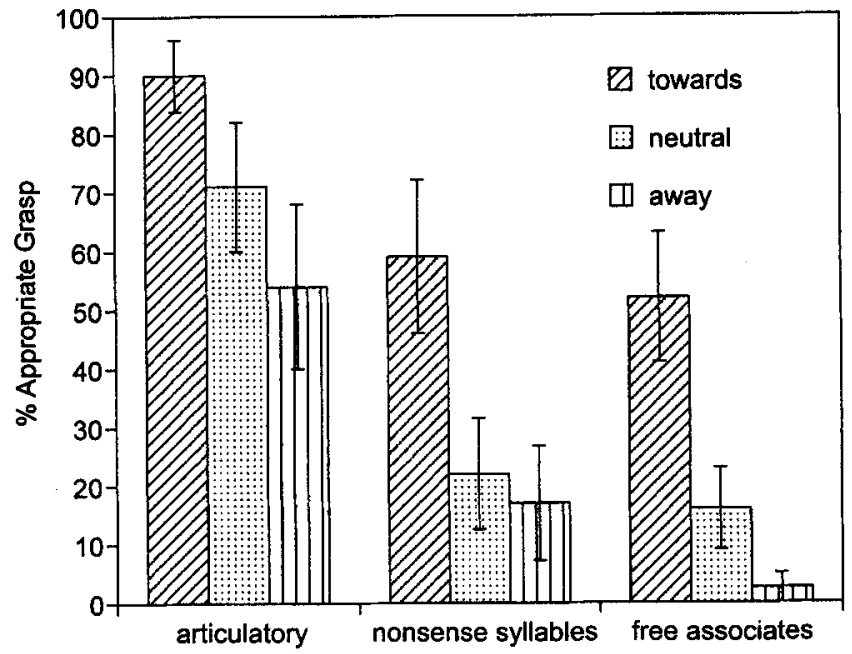

Figure 7. Mean percentages of objects grasped appropriately $( \pm 1 S E)$ as a function of object orientation in Experiment 3.

concurrent task condition that did not involve a difficult memory component. We developed the free-associates task that required participants to retrieve any semantically related word. Furthermore, we asked whether a difficult memory task involving meaningless syllables would also lead to interference. As a third condition, we introduced a classic articulatory suppression task that is known to interfere with the phonological loop subcomponent of working memory (Baddeley, Lewis, \& Vallar, 1984) but does not involve semantic processing or the retrieval of information from long-term memory. We found that grasping was impaired in both the free-associates and nonsense-syllable conditions but not in the articulatory task condition.

An evaluation of concurrent grasping performance in the three distractor task conditions suggests the importance of memory retrieval processes in grasping an object appropriately by its handle. As predicted, performing an "easier" semantic task continued to interfere with appropriate grasping, whereas articulatory suppression, an easy nonsemantic task, did not. However, performing the nonsense-syllable task also led to interference in the grasping task. Although this task most likely involved less semantic processing than the real-word task in Experiment 1, it continued to involve memory retrieval processes as well as a difficult memory component. Tasks that interfered with grasping performance all involved memory retrieval (real-word pairs, nonsense-word pairs, and free associates), whereas tasks that did not interfere involved only spatial or verbal working memory components (the spatial task of Experiment 1 and the articulatory task of Experiment 3). Variations in memory difficulty of the retrieval tasks did not have an effect. We propose that retrieval of semantic information about an object is a necessary component of grasping objects in an appropriate manner. This retrieval can suffer interference as a result of either a semantic or nonsemantic memory retrieval task.

\footnotetext{
${ }^{3}$ As in Experiment 1, mean percentage scores are reported in the text for clarity of presentation.
} 


\section{General Discussion}

Although the visuomotor system can work on its own to guide actions toward objects in space, its independence is limited. Previous studies showed that the action system appears to function independently only when the action is directed at the objects and when the execution of action is not delayed. The present studies illustrate another interaction between cognition and action that arises from the need to conform the grasping response to the identity of objects.

Our first study showed that motoric performance on a task requiring cognitive input could be disnupted by a dual task that engaged semantic processing. When given a semantic interference task, the participants failed to use object information to appropriately grasp hand tools by their handles. In contrast, a spatial imagery task did not impair grasping performance. Experiment 2 further confirmed that the semantic task specifically interfered with the semantic component of the grasping task. When presented with a purely visuospatial motor task, participants did not show impairment under the semantic dual-task condition. Relative to Experiment 1, the results of Experiment 2 were clearly reversed. Visuomotor tracking was impaired by the spatial imagery task but not by the semantic task. Experiment 3 demonstrated differential effects of verbal distractor tasks. Appropriate grasping was reduced with both an "easy" semantic task and a "difficult" pairedassociates task with a reduced semantic component but was not affected by an easy, nonsemantic, non-memory-retrieval task. These results suggest that memory retrieval tasks may interfere with the retrieval and processing of semantic information necessary for appropriate grasping, regardless of the difficulty of the task.

The overall results are consistent with those of recent clinical case studies in which patients with brain injury were observed while grasping tools. On the basis of evidence of DF's ability to guide her actions toward simple objects that do not require processing of phenomenal object characteristics, Carey et al. (1996) tested this visual form agnosic's ability to pick up common objects such as tools and utensils. They presented her with everyday three-dimensional objects and asked her to pick them up with one hand and demonstrate their use. Her performance, relative to that of normal controls, was not impaired in reaching or grasping but was, however, quite different with regard to where she grasped the objects. When the object was presented with the handle facing toward her, she picked it up appropriately, as did the control participants. However, when the handle faced away from her, she grasped the object in an inappropriate place. In all, DF's grasping of the objects was effective but inappropriate for their use.

Testing neurologically intact participants, our present studies introduce several ideas about the nature of action representations. The principal task studied in this research, grasping familiar objects by their handles, required not only a motoric representation of how to grasp the object but also an object-relative representation of where to grasp the object based on its meaningful identity. This distinction is one that Jeannerod (1997) made between pragmatic and semantic representations. For Jeannerod, a pragmatic representation is defined by rapid sensorimotor transformations of the object as a goal for action. In contrast, the semantic representation allows for the integration of object features into a meaningful entity. Although grasping a neutral object, such as a rock, requires only an implicit, pragmatic representation, a number of studies have investigated the ways that semantic representations come into play. Klatzky and colleagues (Klatzky, McCloskey, Doherty, Pellegrino, \& Smith, 1987; Klatzky, Pellegrino, McCloskey, \& Lederman, 1993) have shown that people have explicit knowledge about how to manipulate and grasp objects. For example, Klatzky et al. (1987) found that participants were able to reliably report which class of hand shape (e.g., clench, pinch, poke, or palm) would be used to manipulate a given object and to consistently report a class of objects that could be manipulated when a given hand shape was provided. Similarly, Rosenbaum et al. (1990) found that people adjust their grip patterns on the basis of what they intend to do with the objects being grasped. For example, participants planned an initial grip of a bar in a way that minimized the awkwardness of the final goal position.

\section{Do Perceived Objects Potentiate Actions?}

A number of recent studies have suggested that perceived objects are intimately tied to the actions with which they are associated. Tucker and Ellis (1998) recently suggested that the representation of visual information involves the representation of information about possible actions. Using a stimulus-response compatibility paradigm, they examined whether the orientation of graspable objects would preferentially activate the hand most likely to grasp the object and thus facilitate a keypress response carried out by the same hand. Their task required participants to press a key with their right or left hand depending on whether the picture of an object was upright or inverted. They found that the position of the object's handle had a significant effect on the speed of keypress responses, even though the horizontal position of the object had no relevance to the object-inversion task. For example, handle orientation toward the right facilitated the keypress response made with the right hand. The result that viewing an object in a certain position affected potential for subsequent action suggests that action-related information about objects is represented automatically when an object is viewed.

Whereas it is clear that there is a close connection between visual and motor representations of objects, a question exists as to how an appropriate action on a meaningful object is selected. One theory proposes that there is a direct route from vision to action that bypasses a semantic representation (Riddoch, Humphreys, \& Price, 1989; Rumiati \& Humphreys, 1998). The theory of a direct visual route in the selection of action comes from evidence of double dissociations between abilities of patients with optic aphasia and apraxia. Generally, optic aphasic patients fail to name objects that are presented visually but usually can appropriately gesture as to the use of the object (Campbell \& Manning, 1996). In contrast, apraxic patients can name and recognize objects but fail to gesture appropriately (e.g., Riddoch et al., 1989). Recently, Rumiati and Humphreys (1998) provided evidence for a direct visual route to action in a series of experiments with normal participants. They measured the types of errors made in gesturing and naming pictures or words under time pressure. In a series of experiments, participants were presented with words or pictures and asked to name the object or to gesture as to the use of the object. Errors were classified as "visual" if the incorrect response was related visually (i.e., similar shape) to the presented item. Errors were "semantic" if the response item was functionally, but 
not visually, related to the presented item. In all, Rumiati and Humphreys found that more visual errors were made in gesturing to pictures than to words, and more semantic errors were made in naming pictures than in gesturing. They suggested that the visual errors resulted from the use of a direct route from the visual object to stored actions and that semantic errors resulted from an indirect route from the verbal presentation through semantic knowledge to action.

As an alternative to a direct route from vision to action, other theories suggest that meaningful actions require some access to a conceptual or semantic system (Ochipa, Rothi, \& Heilman, 1989; Rothi, Ochipa, \& Heilman, 1991; Roy, 1983). This view suggests that appropriate use of objects requires semantic processing of the object to inform an action plan. Evidence of patients diagnosed with both optic aphasia and apraxia supports the view that the two share a common semantic network (Endo, Makishita, Yanagisawa, \& Sugishita, 1996). The deficits of these patients suggest that a semantic representation is tied to appropriate use of an object. Buxbaum, Schwartz, and Carew (1997) recently proposed an account of meaningful actions based on a combination of semantic and nonsemantic routes to action. Reviewing patient data, they proposed that a direct nonsemantic route to action exists, but is limited, and that accessing a semantic memory system allows for error-free action.

Studies of neuropsychological patients with apraxia also suggest several accounts of action selection. Patient LL has damage to her posterior parietal lobe. Although she shows normal grasping in the context of simple reaching movements, she is markedly impaired in her grasp in the context of tool use. Sirigu et al. (1995) suggested two possible explanations for this patient's failure to grasp objects in the appropriate way. It may be that the connection between visual-associative areas for semantic recognition and motor processing streams is impaired. LL may be able to recognize the functional utility of the object but cannot use this knowledge to appropriately shape her fingers. Alternatively, Sirigu et al. suggested that LL suffers damage in regard to specialized representations for complex manual postures. This interpretation proposes that motor schemas for hand postures are distinctly represented and directly activated by object features (Iberall \& Arbib, 1990). Sirigu, Duhamel, and Poncet (1991) suggested that there may be neurally dissociable processes for semantic knowledge and affordance-based identification, and they supported this conjecture with evidence from another patient with associative agnosia. This patient could position his hand on an object correctly for use but could not name the purpose of this functional grasp.

Recent neuroimaging studies also suggest neural specificity in the processing of hand tools. A recent study using positron emission tomography (PET) showed that simply observing common tools led to left premotor cortex activation, in the absence of any motoric response (Grafton, Fadiga, Arbib, \& Rizzolatti, 1997). Whereas observing the tools activated dorsal premotor areas, silently naming the tool use activated dorsal and ventral premotor areas as well. In addition, the left inferior frontal sulcus was activated, an area recently found to be involved in the recognition of man-made objects. These findings suggest that premotor activation is specific to the observation of familiar graspable objects. The authors suggest that the dorsal and ventral premotor activations may be a part of the frontotemporal circuits that connect object meaning with motoric response. In a related PET study,
Martin, Wiggs, Ungerlieder, and Haxby (1996) compared naming pictures of tools and naming pictures of animals. The animals minus tools comparison revealed left medial occipital activation, whereas the tools minus animals comparison revealed middle temporal gyrus and left premotor areas. This distributed semantic representation suggests that tools may have a distinct area of representation in the premotor cortex as a result of their functional identity.

That objects are perceived relative to action is not a new concept. Examination of the perception of objects in terms of their use relates to what J. J. Gibson (1979) labeled as object affordances. Gibson wrote that we directly perceive what an object offers for use, rather than its categorical identity. He stated that "to perceive an affordance, is not to classify an object" (Gibson, 1979, p. 134). An object may afford a human action without the classification of what it is. How then do we reconcile this view with our evidence that semantic information about an object appears to inform an individual about where to grasp the object? It seems the distinction can be made between the use of an object in any appropriate way and the use of an object in a way appropriate for its semantic-functional identity. Gibson's account of affordances correctly suggests that an object may afford many uses based on its physical characteristics. However, in circumstances in which an object has a specific functional identity, the present studies suggest that semantic information about that object's function must be retrieved before an object-appropriate action can be taken.

\section{Conclusion}

Evidence from neuropsychological and cognitive research suggests separate systems for phenomenal experience and visuomotor behavior. However, recent research suggests that the independence breaks down under several factors (e.g., time delay or nonegocentric response measures). The present studies illustrate a case of immediate cognitive influence on actions that are directed toward artificial objects such as hand tools. Our results suggest that, without the influence of the cognitive system, the visuomotor system can reach and grasp an object effectively. However, to grasp an object appropriately in a manner defined by its functional identity, at least partial information from the semantic system is needed. These findings illustrate a necessary interaction between visual cognition and visually guided action.

\section{References}

Aglioti, S., DeSouza, J. F. X., \& Goodale, M. (1995). Size-contrast illusions deceive the eye but not the hand. Current Biology, 5, 679-685. Baddeley, A. D., Grant, S., Wight, E., \& Thomson, N. (1975). Imagery and visual working memory. In P. M. A. Rabbitt \& S. Dornic (Eds.), Attention and performance $V$ (pp. 205-217). London: Academic Press. Baddeley, A. D., Lewis, V. J., \& Vallar, G. (1984). Exploring the articulatory loop. Quarterly Journal of Experimental Psychology, 36, 233252.

Bridgeman, B., Peery, S., \& Anand, S. (1997). Interaction of cognitive and sensorimotor maps of visual space. Perception \& Psychophysics, 59, 456-469.

Brooks, L. R. (1968). Spatial and verbal components in the act of recall. Canadian Joumal of Psychology, 22, 349-368.

Buxbaum, L. J., Schwartz, M. F., \& Carew, T. G. (1997). The role of semantic memory in object use. Cognitive Neuropsychology, 14, 219254. 
Campbell, R., \& Manning, L. (1996). Optic aphasia: A case with spared action naming and associated disorders. Brain and Language, 53, 183221.

Carey, D. P., Harvey, M., \& Milner, A. D. (1996). Visuomotor sensitivity for shape and orientation in a patient with visual form agnosia. Neuropsychologia, 34, 329-337.

Creem, S. H., \& Proffitt, D. R. (1998). Two memories for geographical slant: Separation and interdependence of action and awareness. Psychonomic Bulletin \& Review, 5, 22-36.

Dijkerman, H. C., Milner, A. D., \& Carey, D. P. (1998). Grasping spatial relationships: Failure to demonstrate allocentric visual coding in a patient with visual form agnosia. Cognition and Consciousness, 7, 424437.

Endo, K., Makishita, H., Yanagisawa, N., \& Sugishita, M. (1996). Modality specific naming and gesture disturbances: A case with optic aphasia, bilateral tactile aphasia, optic ataxia, and tactile apraxia. Cortex, 32, 3-28.

Franz, V. H., Gegenfurtner, K. R., Bulthoff, H. H., \& Fahle, M. (2000). Grasping visual illusions: No evidence for a dissociation between perception and action. Psychological Science, 11, 20-25.

Gibson, J. J. (1979). The ecological approach to visual perception. Boston: Houghton Mifflin.

Goodale, M. A., Jakobson, L. S., \& Keillor, J. M. (1994). Differences in the visual control of pantomimed and natural grasping movements. Neuropsychologia, 32, 1159-1178.

Goodale, M. A., Jakobson, L. S., Milner, A. D., Perrett, D. I., Benson, P. J., \& Hietanen, J. K. (1994). The nature and limits of orientation and pattern processing supporting visuomotor control in a visual form agnosic. Journal of Cognitive Neuroscience, 6, 46-56.

Goodale, M. A., Meenan, J. P., Bultoff, H. H., Nicolle, D. A., Murphy, K. J., \& Racicot, C. I. (1994). Separate neural pathways for the visual analysis of object shape in perception and prehension. Current Biology, 4, 604-610.

Goodale, M. A., Milner, A. D., Jakobson, L. S., \& Carey, D. P. (1991). A neurological dissociation between perceiving objects and grasping them. Nature, 349, 154-156.

Grafton, S. T., Fadiga, L., Arbib, M. A., \& Rizzolatti, G. (1997). Premotor cortex activation during observation and naming of familiar tools. Neuroimage, 6, 231-236.

Haffenden, A. M., \& Goodale, M. A. (1998). The effect of pictorial illusion on prehension and perception. Journal of Cognitive Neuroscience, 10, $122-136$

Iberall, T., \& Arbib, M. A. (1990). Schemas for the control of hand movements: An essay on cortical localization. In M. A. Goodale (Ed.), Vision and action: The control of grasping (pp. 204-242). Norword, NJ: Ablex.

Jakobson, L. S., Archibald, Y. M., Carey, D. P., \& Goodale, M. A. (1991). A kinematic analysis of reaching and grasping movements in a patient recovering from optic ataxia. Neuropsychologia, 29, 803-809.

Jeannerod, M. (1997). The cognitive neuroscience of action. Oxford, England: Blackwell.

Jeannerod, M., Decety, J., \& Michel, F. (1994). Impairment of grasping movements following a bilateral posterior parietal lesion. Neuropsychologia, 32, 369-380.

Klatzky, R. L., McCloskey, B., Doherty, S., Pellegrino, J., \& Smith, T.
(1987). Knowledge about hand shaping and knowledge about objects. Journal of Motor Behavior, 19, 187-213.

Klatzky, R. L., Pellegrino, J., McCloskey, B. P., \& Lederman, S. J. (1993). Cognitive representations of functional interactions with objects. Memory \& Cognition, 21, 294-303.

Mack, A., \& Rock, I. (1998). Inattentional blindness: Perception without attention. Cambridge, MA: MIT Press.

Martin, A., Wiggs, C. L., Ungerlieder, L. G., \& Haxby, J. V. (1996). Neural correlates of category-specific knowledge. Nature, 379, 649-652.

Milner, A. D., \& Goodale, M. A. (1995). The visual brain in action. Oxford, England: Oxford University Press.

Ochipa, C., Rothi, L. J. G., \& Heilman, K. M. (1989). Ideational apraxia: A deficit in tool selection and use. Annals of Neurology, 25, 190-193.

Pavani, F., Boscagli, I., Benvenuti, F., Rabuffetti, M., \& Farne, A. (1999). Are perception and action affected differently by the Titchner circles illusion? Experimental Brain Research, 127, 95-101.

Proffitt, D. R., Bhalla, M., Gossweiler, R., \& Midgett, J. (1995). Perceiving geographical slant. Psychonomic Bulletin \& Review, 2, 409-428.

Riddoch, M. J., Humphreys, G. H., \& Price, C. J. (1989). Routes to action: Evidence from apraxia. Cognitive Neuropsychology, 6, 437-454.

Rosenbaum, D. A., Marchak, F., Barnes, H. J., Vaughan, J., Slotta, J. D., \& Jorgensen, M. J. (1990). Constraints for action selection: Overhand versus underhand grips. In $\mathrm{M}$. Jeannerod (Ed.), Attention and performance XIII (pp. 321-342). Hillsdale, NJ: Erlbaum.

Rossetti, I. (1998). Implicit short-lived motor representation of space in brain-damaged and healthy subjects. Consciousness and Cognition, 7 , $520-558$.

Rothi, L. J. G., Ochipa, C., \& Heilman, K. M. (1991). A cognitive neuropsychological model of limb praxis. Cognitive Neuropsychology, 8, 443-458.

Roy, E. A. (Ed.). (1983). Neuropsychological perspectives on apraxia and related action disorders. Amsterdam: North-Holland.

Rumiati, R. I., \& Humphreys, G. W. (1998), Recognition by action: Dissociating visual and semantic routes to action in normal observers. Jourmal of Experimental Psychology: Human Perception and Performance, 24, 631-647.

Sirigu, A., Cohen, L., Duhamel, J., Pillon, B., Dubois, B., \& Agid, Y. (1995). A selective impairment of hand posture for object utilization in apraxia. Cortex, 31, 41-55.

Sirigu, A., Duhamel, J.-R., \& Poncet, M. (1991). The role of sensorimotor experience in object recognition. Brain, 114, 2555-2573.

Tucker, M., \& Ellis, R. (1998). On the relations between seen objects and components of potential actions. Journal of Experimental Psychology: Human Perception and Performance, 24, 830-846.

Tukey, J. W. (1977). Exploratory data analysis. Reading, MA: AddisonWesley.

Welch, R. B., Post, R. B., Lum, W., \& Cohen, M. M. (1996, November). Are vision and action dissociated with respect to pictorial illusions? Paper presented at the meeting of the Psychonomic Society, Chicago, IL.

Willingham, D. B., Hollier, J., \& Joseph, J. (1995). A Macintosh analogue of the rotary pursuit task. Behavior Research Methods, Instruments, \& Computers, 27, 491-495.

Wraga, M., Creem, S. H., \& Proffitt, D. R. (2000). Perception-action dissociations of a walkable Muller-Lyer configuration. Psychological Science, 11, 239-243. 
CREEM AND PROFFITT

\section{Appendix}

Word Lists Used in the Present Experiments

Paired-associates word list

1. Celery-pear

2. Rug-lamp

3. Cookie-soup

4. Tomato-orange

5. Butter-peas

6. Radio-table

7. Television-desk

8. Bed-cabinet

9. Bird-fish

10. Dog-mouse

11. Computer-chair

12. Whale-raccoon

13. Frog-penguin

14. Cheese-bread

15. Tiger-skunk
16. Squirrel-elephant

17. Onion-peach

18. Cracker-melon

19. Doctor-salesman

20. Teacher-gardener

21. Cat-bumblebee

22. Pineapple-cucumber

23. Sweater-glasses

24. Coat-earrings

25. Trumpet-piano

26. House-phone

27. Pillow-curtains

28. Window-vase

29. Apple-broccoli

30. Lettuce-banana

Nonsense-syllable word list

1. ZIJ-KIJ

2. KUJ-MUJ

3. GEC-YEC

4. ZOF-VOF

5. BOJ-MOJ
6. YEF-VEF

7. KIF-YIF

8. YIX-GIX

9. YUB-VUB

10. LAJ-ZAJ

Received August 17, 1999

Revision received February 22, 2000

Accepted May 17, 2000 